\title{
Experimental Characterization of Spray generated by a Rotary Atomizer Wheel
}

\author{
Maximilian Kuhnhenn*1, Max Frederik Luh" ${ }^{1}$, Tórstein V. Joensen², Ilia V. Roisman ${ }^{1}$, Cameron \\ Tropea $^{1}$ \\ ${ }^{1}$ Institute for Fluid Mechanics and Aerodynamics, Technische Universität Darmstadt, \\ Darmstadt, Germany \\ ${ }^{2}$ GEA Process Engineering A/S, Soeborg, Denmark \\ ${ }^{*}$ Corresponding author: Kuhnhenn@sla.tu-darmstadt.de
}

\begin{abstract}
Rotary atomizers are widely used for spray drying processes to produce powders in various industrial applications. The atomization regime and therefore the droplet size distribution have a substantial impact on the process outcome. Depending on the intended use of the dried powder particles, various droplet size distributions are desired, which should be adjustable by the main operating parameters (e.g. mass flow rate and wheel speed). In this study the spray of a rotary atomizer wheel, operated under normal ambient conditions, is characterized for different mass flow rates, wheel speeds and viscosities with the help of a phase Doppler measurement system. Visual investigations of the spray with a high resolution camera system help to get a better understanding of the atomization process, as well as the thickness of the liquid jets exiting the atomizer.
\end{abstract}

\section{Keywords}

rotary atomizer, spray drying, phase Doppler analyser

\section{Introduction}

The intended use of the dried powder obtained with a rotary atomizer in a spray drying process [1] or for flue gas cleaning [2] determines the desired size distribution of the powder particles, their morphology and porosity. It is important to understand the influencing parameters on the droplet size distribution for a given geometry of an atomizer to be able to change them to achieve a desired droplet size distribution.

In rotary atomizers the liquid is first supplied to the internal atomizer reservoir, flows from there into several (ceramic-) inserts (mainly driven by the centrifugal force due to the atomizer rotation) and is then ejected into the surrounding air. The jets of the processed liquid then breakup and atomize into drops with a certain size distribution. Besides the spray drying industry $[3,4,5]$ rotary atomizers $[6,7]$ and ligament based atomization is also used in numerous other applications, e.g. painting, coating, and generation of agricultural sprays (spreading of pesticides) [8]. The primary atomization process for laminar operating rotary atomizers has already been expressed in terms of dimensionless numbers [9, 10], but such atomizers operate at much lower rotational speeds and mass flow rates compared to the atomizers investigated in this study.

\section{Experimental Method}

The experiments in this study are conducted with two different experimental setups, as shown in Fig. 1. In both setups a rotary atomizer wheel, a liquid supply system and a spray trap, to catch the atomized liquid, are used. The atomizer is operated at $10,5001 / \mathrm{min}$ to $29,0001 / \mathrm{min}$ and the feed rate is varied between $50 \mathrm{~kg} / \mathrm{h}$ to $450 \mathrm{~kg} / \mathrm{h}$. The processed liquids are water (viscosity $\eta=1 \mathrm{mPas}$ ) and two different aqueous glycerol water solutions (viscosity $\eta=20 \mathrm{mPa}$ s and $\eta=50 \mathrm{mPa}$ ). The first experimental setup (cf. Fig. 1(a)) comprises a high resolution imaging system to observe the atomization process and to (optically) measure the thickness of the liquid jet exiting the atomizer wheel. For further investigations of the atomization a phase Doppler (PD) system is used, as shown in Fig. $1(b)$

The high resolution imaging system consists of a pco-edge 5.5 camera (resolution: $2560 \mathrm{px} \times 2160 \mathrm{px}, 30 \mathrm{~Hz}$ ), a Nikon AF Micro Nikkor lens with $105 \mathrm{~mm}$ focal length, a DualPower 200-15 laser system with 6 ns pulse length and a ShadowStrobe optics to illuminate the diffusing plate by using a mirror. The laser is capable of generating laser pulses at $15 \mathrm{~Hz}$ per cavity and therefore only every 6th to 16th revolution can be captured. Nevertheless, this low frequency is not an obstacle for the collection of a large number of images to achieve statistical significance. The camera is operated in global shutter mode and, due to the short illumination time (6 ns), no motion blur is observed, although the speed of the wheel can reach up to $150 \mathrm{~m} / \mathrm{s}$. The field of view (FOV) is $17.5 \mathrm{~mm} \times 14.8 \mathrm{~mm}$, resulting in a resolution of $6.85 \mu \mathrm{m} / \mathrm{px}$.

The PD system (cf. Fig. 1(b)) is operated in the dual-mode configuration to measure droplet size and velocity distributions of the resulting spray [11]. By adjusting the orientation of transmitting and receiving probe the tangential and radial velocity of the spray exiting the atomizer wheel can be measured. The gravitational influence and the resulting velocity component is neglected, as the spray is oriented horizontally. The PD system can be moved laterally in a range of $30 \mathrm{~mm}$ to $190 \mathrm{~mm}$ distance to the wheel, to be able to capture different stages of the atomization process. The main settings of the system, used to acquire the data presented in this study, are summarized in Table 1. As the atomizer wheel has 4 inserts that pass by the measurement volume of the PD system, the measured spray is 
(a)

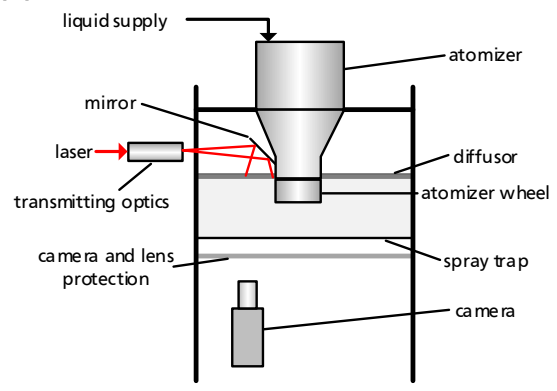

(b)

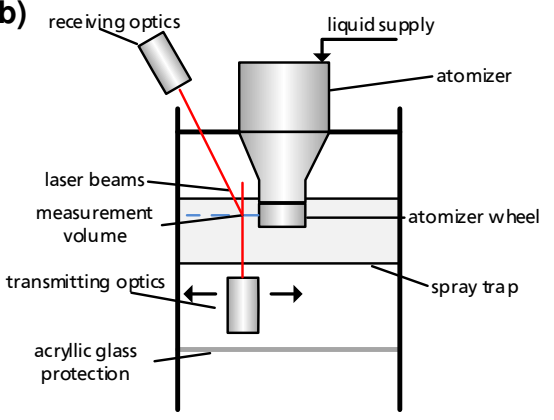

Figure 1. (a) Schematic of experimental setup including: liquid supply system, atomizer and high-resolution imaging system. (b) Alternative configuration with the laser spray diagnostic system (PD) to measure droplet size and velocity. The PD system can be moved laterally using a traversing system to perform measurements at different positions.

highly unsteady. An encoder signal from the atomizer (one signal per revolution) is used as a reference signal for the PD measurements. The detected droplets are assigned to the reference signal in time, which makes it possible to conduct angular resolved measurements (phase averaged with $0.5^{\circ}$ resolution).

Table 1. Settings of the phase Doppler measurement system

\begin{tabular}{lr}
\hline Parameter & Setting \\
\hline Laser Power & $60 \mathrm{~mW}$ \\
Wavelength 1 & $488 \mathrm{~nm}$ \\
Wavelength 2 & $514.5 \mathrm{~nm}$ \\
Transmitter focal length & $600 \mathrm{~mm}$ \\
Receiver focal length & $500 \mathrm{~mm}$ \\
Scattering angle & $30^{\circ}$ \\
Refractive index water & 1.3330 \\
Refractive index 20 mPa s & 1.4255 \\
Refractive index 50 mPa s & 1.4401 \\
Aperture mask & Mask A \\
Maximum particle diameter & $215.2 \mu \mathrm{m}$ \\
Phase validation ratio & $15 \%$ \\
Slit width & $100 \mu \mathrm{m}$
\end{tabular}

\section{Mechanisms of observed Atomization}

The atomization is observed with the high resolution imaging system (cf. Fig. 1(a)) and shown exemplary for one operating point and different viscosities in Fig. 2. The angular velocity and feed rate are kept constant from Fig. 2(a) to 2(c). The processed liquid exits the atomizer wheel through the ceramic insert in a jet type flow, where most of the liquid is accumulating on the trailing edge. This effect is caused by the Coriolis force acting on the liquid. The Coriolis force is acting perpendicular to the main (radial) jet velocity and, by considering the vector of rotation, the liquid is forced towards the trailing edge of the insert (cf. Fig. 2). In addition to the main jet at the trailing edge, the liquid exits the insert in an annular film flow, wetting the hole periphery of the insert. At a viscosity of $\eta=1 \mathrm{mPa}$ s the thin annular film breaks up immediately after the exit (cf. Fig. 2(a)), whereas an increasing viscosity provokes small fingers, protruding from the thin annular film (cf. Fig. 2(b)). These small fingers get longer with a further increase in viscosity from $\eta=20 \mathrm{mPa}$ s to $50 \mathrm{mPa}$ s, as shown in Fig. 2(c). Increasing viscosity leads to a higher mass flow through the thin annular film and a decreasing mass flow through the main jet, while the total mass flow remains constant. Based on these initial photographic images of the atomization process, it is evident that the atomization changes with different viscosities.

\section{Airflow characterization}

To better understand the mechanisms of atomization the velocity field around the wheel has to be characterized in detail. As the wheel is rotating with a high angular velocity and the ambient air in the far field is quiescent, a boundary layer develops. The tangential velocity of the wheel can be calculated by

$$
\hat{u}_{t}=\omega R_{a} .
$$

The tangential velocity of the wheel varies from $55 \mathrm{~m} / \mathrm{s}$ to $150 \mathrm{~m} / \mathrm{s}$, depending on the angular velocity of the wheel. As the wheel has radial openings and is not encapsulated from the surroundings, air is accelerated through the radial openings, based on the centrifugal force (even without water). Pipe friction causes a slight reduction of the 

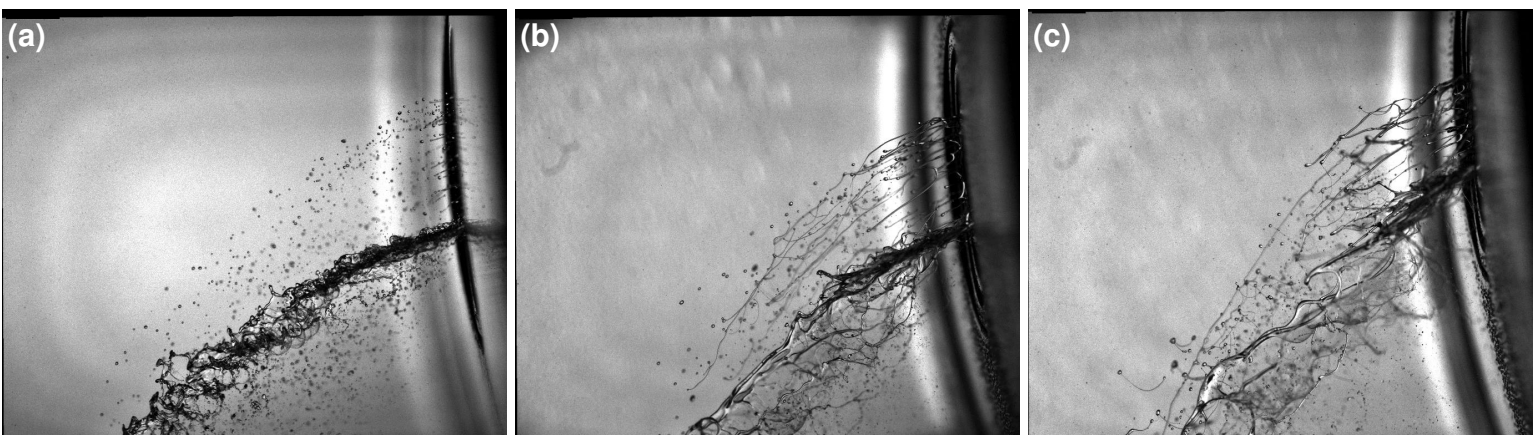

Figure 2. Photographic images of the liquid exiting the atomizer in a jet shape. The speed of the wheel is $10,5001 / \mathrm{min}$ and the feed rate is $100 \mathrm{~kg} / \mathrm{h}$. (a) Viscosity $\eta=1 \mathrm{mPa}$, (b) Viscosity $\eta=20 \mathrm{mPa} \mathrm{s}$, (c) Viscosity $\eta=50 \mathrm{mPa}$ s

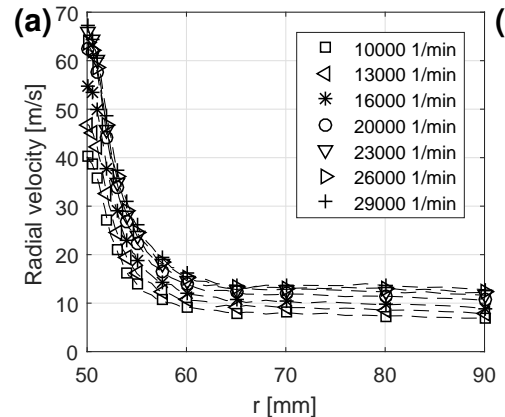

Figure 3. Results of hot-wire measurements.
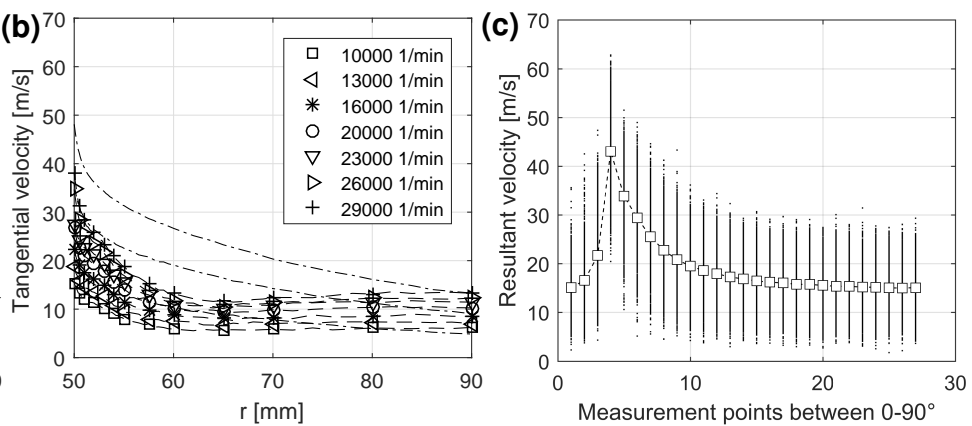
(a) Radial air velocity. (b) Tangenti
with scatter band for $10,5001 / \mathrm{min}$.

(c) Angular resolved air velocity

resulting air velocity. The radial air velocity can be estimated as:

$$
u_{r}=\sqrt{2 \omega^{2} R_{a} l-\frac{\lambda u_{r}^{2} l}{d}}
$$

This boundary-layer flow and the radial air velocity have a significant impact on the atomization of the resulting spray, which is why further knowledge of the airflow is required. Hot-wire measurements are conducted with a single wire boundary-layer probe (55P14), to capture the evolution of the boundary layer of the pure air flow. The experiments are conducted with a StreamLine Pro CTA System from Dantec Dynamics, $20 \mathrm{kHz}$ sampling rate, a 15 point calibration between $2 \mathrm{~m} / \mathrm{s}$ to $60 \mathrm{~m} / \mathrm{s}$ and 84 measurement positions from $0.5 \mathrm{~mm}$ to $101 \mathrm{~mm}$. The measured air velocity is shown in Figure 3, already split into radial (Fig. 3(a)) and tangential (Fig. 3(b)) velocity components. The decomposition of the raw hot-wire signal was done with the help of Figure 3(c), showing the mean trend of 50,000 acquired samples for one distance and angular velocity, split into orthogonal components, as the flow is periodic due to the four inserts. Figure 3(c) shows the resultant air velocity: the tangential air velocity is represented by the lower part and the peak shows the vector addition of the radial and tangential velocities. Figure 3(a) shows the strong deceleration of the radial air velocity within $10 \mathrm{~mm}$ from the wheel outer surface. Different angular velocities have a high impact on the exit velocity, but with increasing distance this impact rapidly reduces. The decay of the tangential velocity is shown in Figure $3(\mathrm{~b})$. Additional measurements are conducted $10 \mathrm{~mm}$ below the inserts in order to show the influence of the airflow coming out of the inserts, represented by the dashed-dotted lines. In contrast to the tangential velocity in front of the inserts, these results illustrate a different behaviour - the airflow prevents the proper evolution of a boundary layer. In the spray drying process, the airflow around the wheel is influenced by the atomized liquid, but nevertheless these measurements demonstrate the thinness of the boundary layer around the wheel. Even $0.5 \mathrm{~mm}$ away from the wheel the tangential air velocity is far away from the tangential velocity of the wheel itself (cf. Eq. (1)). Therefore a large relative velocity between droplets and the surrounding air is established, leading to a strong deceleration of the atomized liquid.

Figure 4 shows the comparison between the experimentally (CTA) and analytically obtained air velocities (Eq. (1) and (2)). Obviously the theoretical prediction of the tangential velocity is much higher compared to the experiments, showing that already $0.5 \mathrm{~mm}$ away from the wheel the air is strongly decelerated. The deviation of the radial air velocity is much smaller, but increasing with the wheel speed. While theoretical prediction and experiments of $u_{\mathrm{r}}$ match quite well at $10,5001 / \mathrm{min}$, they deviate from each other up to a factor of two at $29,0001 / \mathrm{min}$. This effect can be explained by the insufficient temporal resolution of the experimentally obtained radial insert (air) velocity $u_{\mathrm{r}}$. For example at $29,0001 / \mathrm{min}$ only 10 measurement points can be acquired for $90^{\circ}$, due to the $20 \mathrm{kHz}$ sampling rate (cf. Fig. 3(c)). Furthermore this can lead to an underestimation of the actual air velocity. 


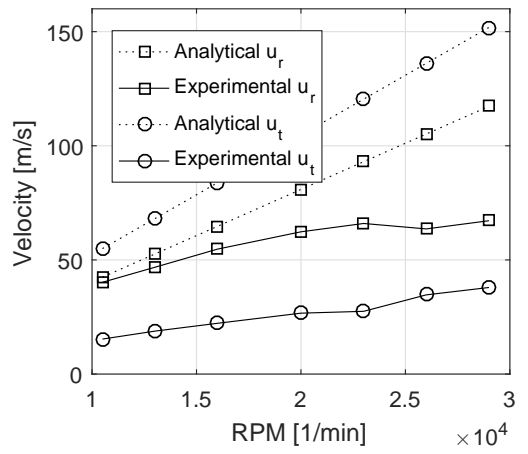

Figure 4. Comparison of analytical and experimental values for radial $\left(u_{\mathrm{r}}\right)$ and tangential $\left(u_{\mathrm{t}}\right)$ air velocities.

\section{Spray characterization}

To characterize the atomization process and the resulting spray, PD measurements are conducted. As the PD system in dual-mode configuration can only measure almost spherical droplets and not a liquid jet, the measurements are conducted beyond the primary breakup zone. From a point $30 \mathrm{~mm}$ radially from the wheel, data is acquired every further $20 \mathrm{~mm}$. Either 50,000 samples or at least $60 \mathrm{~s}$ of measurement time are recorded. Due to the inhomogeneous spray and the liquid jets in the vicinity of the wheel, the validation is poorer compared to the validation in the homogeneous spray, as shown in Fig. 5. With increasing distance, the validation increases and reaches up to $90 \%$. Higher viscosities hinder the measurements in the vicinity of the wheel, as longer ligaments are produced (cf. Fig. 2(c)). Furthermore, it is difficult to measure operating points with $50 \mathrm{~kg} / \mathrm{h}$ mass flow rate, due to the distribution of low volume of atomized liquid in the observation zone and the resulting low data rate combined with high noise levels. The spherical validation (cf. Fig. $5(\mathrm{~b})$ ) is above $70 \%$ in all cases.
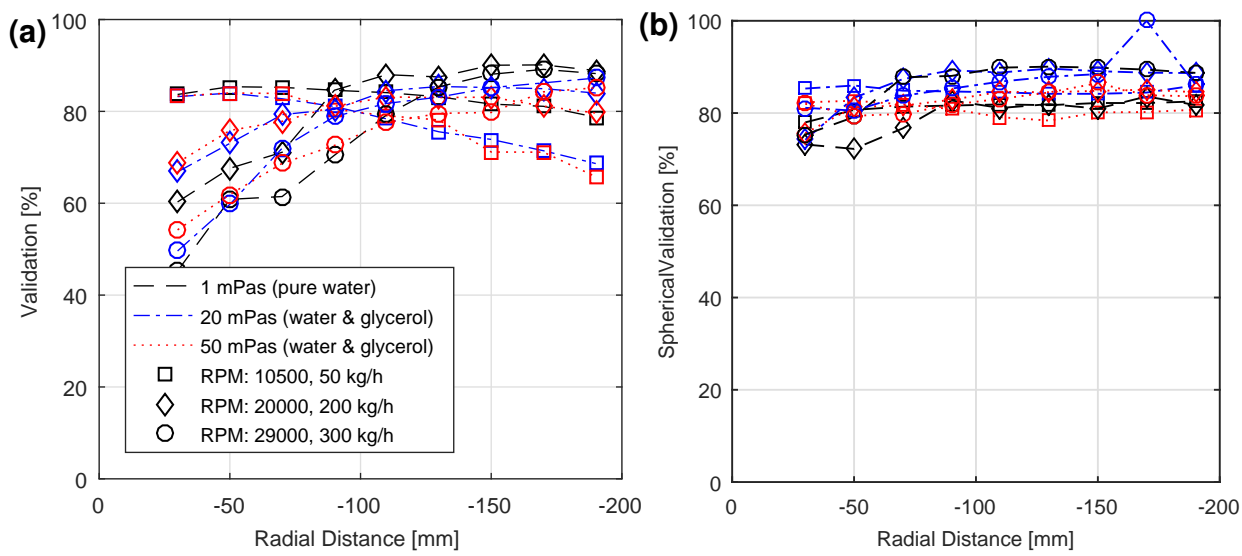

Figure 5. Validation of the conducted PD measurements, depending on the measurement position. (a) Different mass flow rates, viscosities and positions are compared. (b) In all cases the spherical validation is above $70 \%$.

Figure 6 shows the angular resolved velocity components (tangential and radial) of the spray at certain distances from the wheel. Beginning in the vicinity of the wheel, four distinct velocity peaks are visible, reflecting the four inserts of the wheel. As expected, the velocity components decrease with an increase in distance to the wheel (Fig. 6 left to right) due to aerodynamic drag. The tangential velocity component flattens out faster than the radial component, as the initial tangential velocity is lower compared to the radial (also compare to CTA measurements in Fig. 3). As the initial tangential velocity $\hat{u}_{t}$ of the liquid at the exit of the wheel has to match the speed of the wheel, we observe a drastic decrease in tangential velocity, as already seen before. This again shows, that the boundary layer around the rotating wheel must be rather thin.

Figure 7 illustrates the corresponding angular resolved droplet sizes for different radial distances. The mean diameter per angle bin is highlighted by the white markers. This plot indicates, that one mean diameter per measurement point drastically underestimates the droplet size of the jets exiting the wheel. Obviously the droplets produced by the four jets are much bigger compared to the annular thin film, and secondary droplets that are already broken up. With increasing distance, the spray becomes more and more homogeneous and the mean diameter per angle bin increases. Combined with the strong velocity size correlation, this is either a sign of coalescence or of ongoing breakup of filaments that are not recognized by the PD system in the vicinity of the wheel.

To illustrate the influence of wheel speed and mass flow rate on the resulting spray, the Sauter mean diameter $D_{32}$ is plotted in Figure 8 for different viscosities at $110 \mathrm{~mm}$ distance. The influence of the mass flow rate on the $D_{32}$ is rather small, compared to the wheel speed. This behaviour is very interesting for the spray drying industry, as 

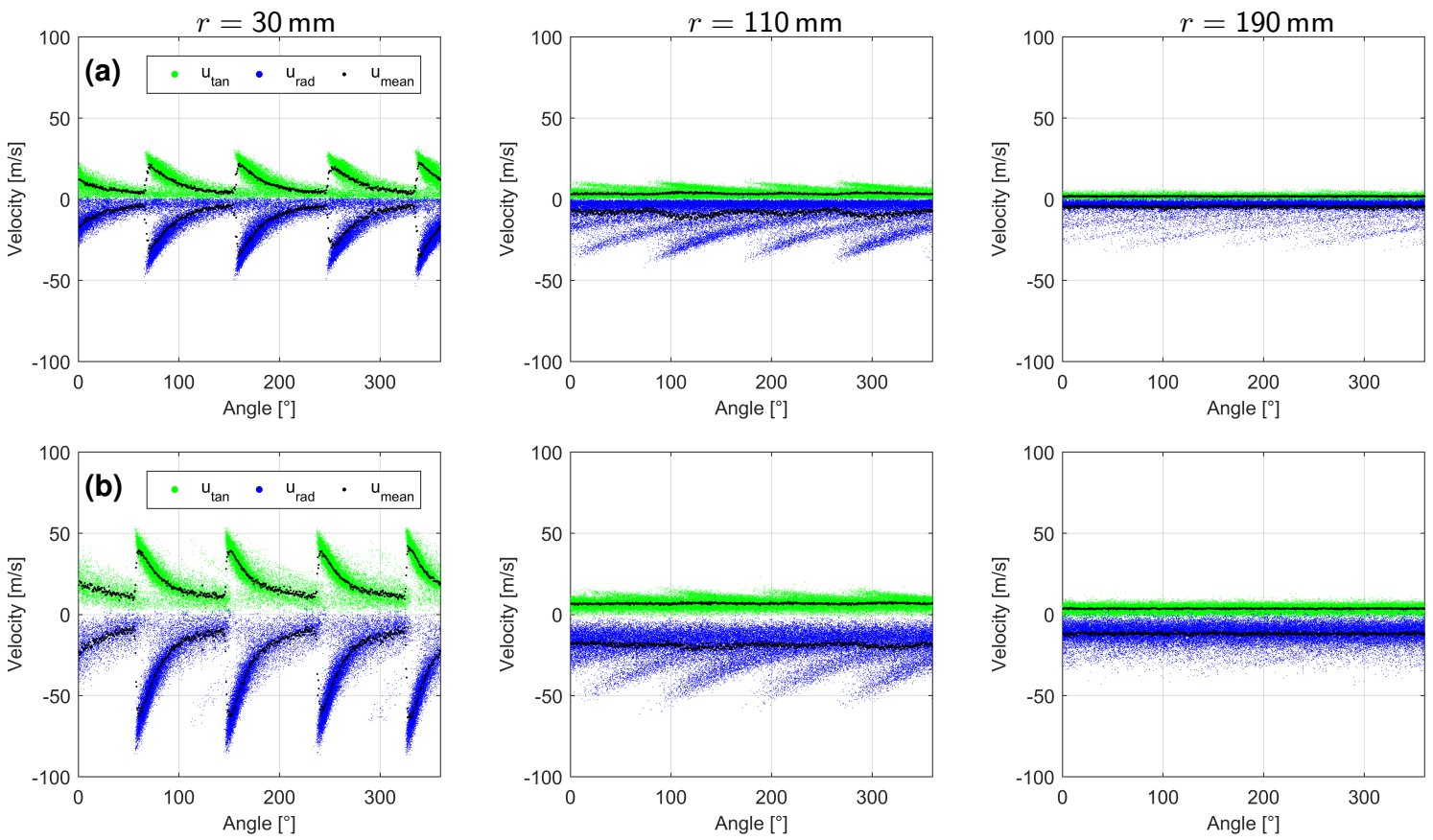

Figure 6. Angular resolved velocity of the resulting spray at different distances to the atomizer exit. The wheel is running with (a) $10,5001 / \mathrm{min}, 50 \mathrm{~kg} / \mathrm{h}$ and $\eta=1 \mathrm{mPa}$; (b) $20,0001 / \mathrm{min}, 200 \mathrm{~kg} / \mathrm{h}$ and $\eta=1 \mathrm{mPa}$ s.
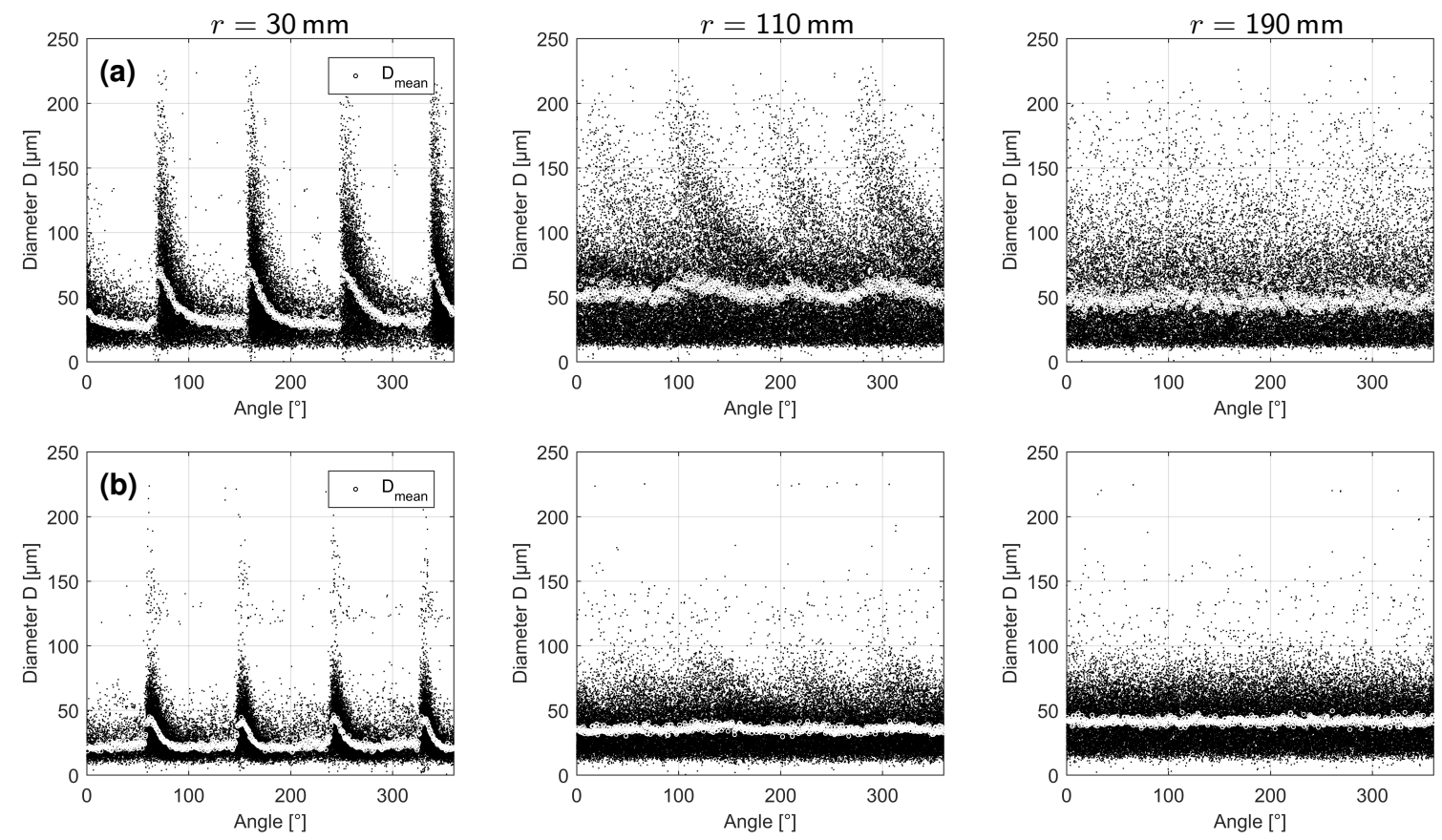

Figure 7. Angular resolved droplet size of the resulting spray at different distances to the atomizer exit. Mean values per angle bin are highlighted by the white markers. The wheel is running with (a) $10,5001 / \mathrm{min}, 50 \mathrm{~kg} / \mathrm{h}$ and $\eta=1 \mathrm{mPa} \mathrm{s}$; (b) $20,0001 / \mathrm{min}$, $200 \mathrm{~kg} / \mathrm{h}$ and $\eta=1 \mathrm{mPa}$. 

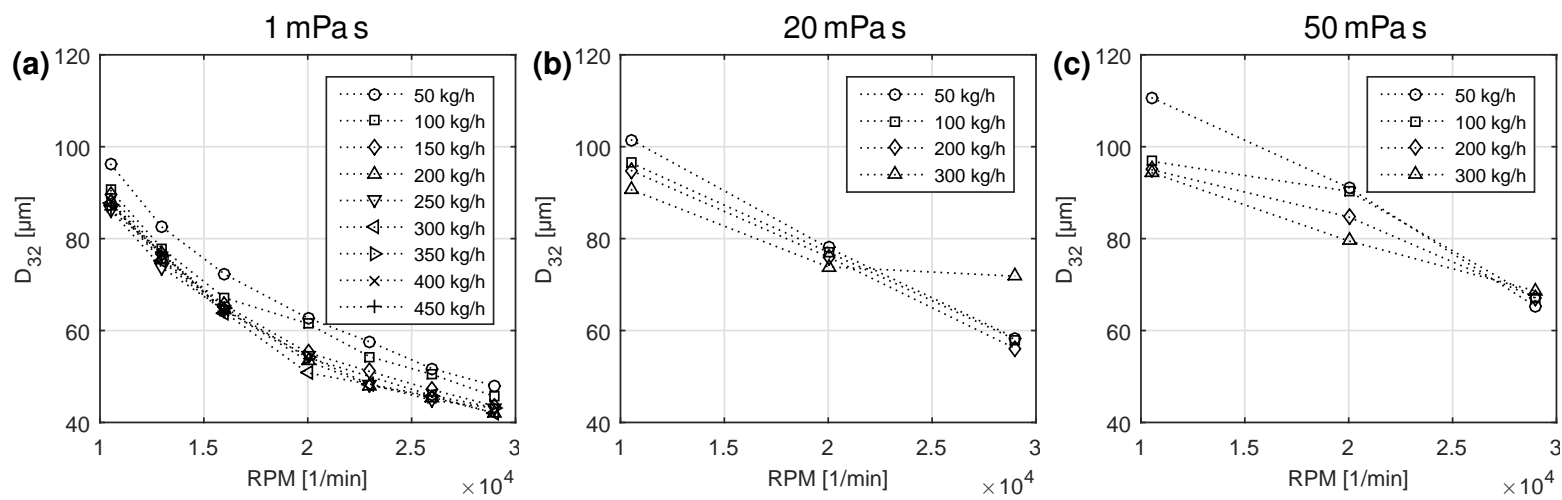

Figure 8. Resulting $D_{32}$ of the spray at a distance of $r=110 \mathrm{~mm}$ to the wheel, where the spray is homogeneous (cf. Figure 7). (a) $\eta=1 \mathrm{mPa}$, (b) $\eta=20 \mathrm{mPas}$, (c) $\eta=50 \mathrm{mPas}$

a change in mass flow rate has a rather small impact on the resulting mean powder size. The main influencing parameter is the speed of the wheel, which can be changed very easily. With increasing viscosity (Fig. 8(a) to 8(c)) the $D_{32}$ is increasing. The negligibly small influence of the mass flow rate still persists.

To further characterize the spray, the width of the volume distribution can be described by the relative span $\Delta$. The relative span $\Delta$ is a dimensionless value for the dispersion of drop sizes, where $\Delta \ll 1$ describes a mono-disperse spray:

$$
\Delta=\frac{D_{0.9}-D_{0.1}}{D_{0.5}}
$$

with the volume mean diameter $D_{0.5} . D_{0.9}$ and $D_{0.1}$ describe the drop size, where $90 \%$ of the drop volume, and $10 \%$ respectively, are contained in droplets of smaller diameters. Figure 9 illustrates the evolution of the relative span $\Delta$ for different wheel speeds (divided into zones of constant speed by the vertical black lines). Within one zone the mass flow rate increases from left to right and several distances are shown for clarity. With increasing wheel speed the relative span $\Delta$ decreases, demonstrating a more uniform droplet size distribution (width of the droplet size distribution histogram). For higher speeds (zones to the right) the relative span $\Delta$ increases with mass flow rate for distances further away, showing a broader distribution. For low mass flow rates and high wheel speeds (e.g. MP 37) the span $\Delta$ is rather broad, showing a change in uniformity with increasing distance - the spray is homogeneous further away. Figure 9 tries to capture several parameters and its influence on the width of the droplet size distribution. It is rather difficult to characterize a spray with only two single numbers $\left(D_{32}\right.$ and $\Delta$ ), but it is evident that the main influencing parameters are the speed of the wheel and the distance from the wheel.

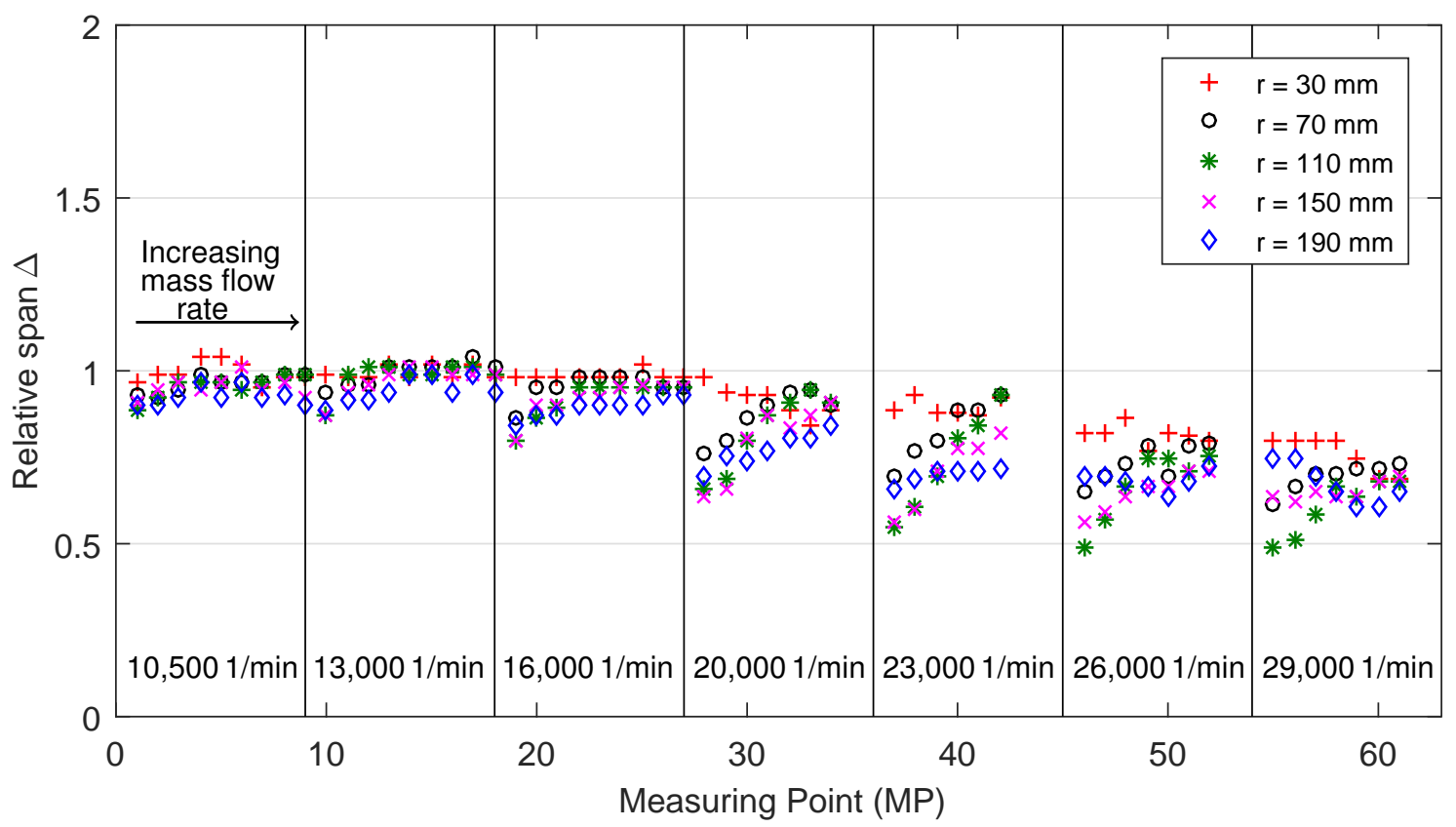

Figure 9. Relative span $\Delta$ (cf. Eq. (3)) for different speeds, mass flow rates and distances to the atomizer exit. The vertical lines divide the diagram into zones of constant speed $(10,5001 / \mathrm{min}$ to $29,0001 / \mathrm{min}$ from left to right). Within one zone the mass flow rate is increasing from $50 \mathrm{~kg} / \mathrm{h}$ to $450 \mathrm{~kg} / \mathrm{h}$ (left to right) in steps of $50 \mathrm{~kg} / \mathrm{h}$. Furthermore different distances to the atomizer wheel are shown to illustrate the ongoing atomization process. 


\title{
Conclusions
}

The ejection of processed liquid and the atomization process have been experimentally investigated in this study. High resolution images of the atomization process clearly show the influence of the viscosity on the formation of ligaments. Furthermore, the importance of the Coriolis force and the resulting jet shape flow of the ejected liquid is observed. Together with PD measurements, the atomization process can be characterized. In the vicinity of the wheel: the boundary layer around the wheel and the resulting strong velocity gradients have a strong impact on the atomization, as high relative velocities act on the drops. The radial oriented inserts in the atomizer wheel produce a radial velocity component, which cannot be neglected. This radial velocity component protrudes further into the surrounding air, than the tangential component, as also shown by the PD measurements. The tangential homogeneity of the spray (in terms of velocity) is reached much faster (closer to the wheel), compared to the radial homogeneity. Although the mean velocities per angle bin already show a homogeneous behaviour at e.g. $110 \mathrm{~mm}$ (cf. Fig.6), the raw values indicate that the mean value doesn't represent the behaviour of the entire spray. Furthermore, measurements conducted without the encoder, leading to one single mean velocity or diameter per measurement point, would lead to a complete missinterpretation of the results - the strong influence of the single jets would be neglected. The variation of diameters near to the wheel is comparably high (e.g. $0 \mu \mathrm{m}$ to $215 \mu \mathrm{m}$ ), but flattens out - depending on the wheel speed and mass flow rate. Additionally, signs of coalescence could be observed, as the mean diameter increases with increasing distance to the wheel, together with a strong deceleration of the flow. The main influencing parameter was found to be the speed of the wheel and not the mass flow rate, as the resulting $D_{32}$ changes drastically with the speed. The influence of viscosity of the processed liquid is - in agreement with the expectations after analysing the initial pictures of the atomization - relatively strong. An increase in viscosity leads to an increase in the resulting mean droplet diameter. The weak influence of the mass flow rate persists. The uniformity of the droplet size distribution was also analysed and it could be shown, that the relative span $\Delta$ only deviates for high wheel speeds. Low mass flow rates lead to a more uniform droplet size distribution, which could not have be seen from the analysis of $D_{32}$ alone.

\author{
Nomenclature \\ $\hat{u}_{t} \quad$ tangential velocity [m/s] \\ $u_{r} \quad$ radial velocity $[\mathrm{m} / \mathrm{s}]$ \\ $\omega \quad$ angular velocity $[1 / \mathrm{s}]$ \\ $R_{a} \quad$ radius of the atomizer wheel [mm] \\ $l \quad$ length of insert [mm] \\ $d \quad$ diameter of insert [mm] \\ $\lambda \quad$ Darcy friction factor [-]
}

\section{References}

[1] GEA Process Engineering A/S, 2016. GEA Niro Spray Drying Absorption - The easy way to clean the flue gas from waste incinerators. http://www.gea.com/global/en/binaries/GEA\%20Spray $\% 20$ drying\% 20absorption\%20Waste\%20Incinerator_tcm11-24419.pdf ([online]. [cit. 2016-01-29]).

[2] GEA Process Engineering A/S, 2016. http://www.gea.com ([online]. [cit. 2016-01-29]).

[3] Mujumdar, A. S., 2014. "Handbook of Industrial Drying". CRC Press, Boca Raton.

[4] Baker, C., 1997. "Industrial Drying of Foods". Springer Science \& Business Media, Heidelberg.

[5] Masters, K., 1979. "Spray Drying Handbook". G. Godwin ; Halsted Press, London; New York, 3. ed.

[6] Ashgriz, N., 2011. "Handbook of Atomization and Sprays: Theory and Applications". Springer US, New York.

[7] Liu, H., 2000. "Science and Engineering of Droplets: Fundamentals and Applications". Noyes Publications Andrew, Park Ridge, NJ Norwich, NY.

[8] Domnick, J., 2012. Sep. 2.-6. 2012, 12th Triennial International Conference on Liquid Atomization and Spray Systems, Heidelberg, Germany.

[9] Walzel, P., Schaldach, G., and Wiggers, H., 2008. Sep. 8.-10. 2008, 22nd European Conference on Liquid Atomization and Spray Systems, Como Lake, Italy.

[10] Mescher, A. and Walzel, P., 2010. Sep. 6.-8. 2010, 23rd Annual Conference on Liquid Atomization and Spray Systems, Brno, Czech Republic.

[11] Tropea, C., Xu, T.-H., Onofri, F., Géhan, G., Haugen, P., and Stieglmeier, M., 1996. Particle \& Particle Systems Characterization, 13(2), pp. 165-170. URL http://dx.doi.org/10.1002/ppsc.19960130216. 